\title{
ON SOME CONDITIONS FOR NONVANISHING OF DETERMINANTS
}

\section{A. M. OSTROWSKI ${ }^{1}$}

1. Among the criteria for nonvanishing of the determinant $D$ of the matrix $A\left(a_{\mu \nu}\right)(\mu, \nu=1, \cdots, n)$, those of particular interest are the ones which use only the moduli $\alpha_{\mu \nu}=\left|a_{\mu \nu}\right|$ of the elements and some simple combinations of these moduli. The most famous is given by the so-called Hadamard's Theorem ${ }^{2}$ that $D \neq 0$ if we have

$$
\alpha_{\mu \mu}>\sum_{\nu \neq \mu} \alpha_{\mu \nu} \quad(\mu=1, \cdots, n) .
$$

Later we gave different criteria of this type using in particular the expressions

$$
\begin{aligned}
& R_{\mu}=\sum_{\alpha \neq \mu} \alpha_{\mu \kappa} \\
& C_{\mu}=\sum_{\alpha \neq \mu} \alpha_{\kappa \mu} \\
& (\mu=1, \cdots, n) \\
& R_{\mu, s}=\left(\sum_{\alpha \neq \mu} \alpha_{\mu \alpha}^{*}\right)^{1 / s}, \quad C_{\mu, s}=\left(\sum_{\alpha \neq \mu} \alpha_{\alpha \mu}^{*}\right)^{1 / s} \quad(\mu=1, \cdots, n), \\
& m_{\mu}=\operatorname{Max}_{\kappa \neq \mu} \alpha_{\mu \kappa}=R_{\mu, \infty}, m_{\mu}^{\prime}=\operatorname{Max}_{\kappa \neq \mu} \alpha_{\kappa \mu}=C_{\mu, \infty}(\mu=1, \cdots, n) .
\end{aligned}
$$

2. An essential link towards the main result of this note was given in 1951 in a previous communication, ${ }^{8}$ namely that for any fixed $\alpha, 0<\alpha<1$, the inequalities

$$
R_{\mu}^{\alpha} C_{\mu}^{1-\alpha}<\alpha_{\mu \mu}, \quad(\mu=1, \cdots, n)
$$

are sufficient. Some special cases of this with $\alpha=1 / 2$, were found earlier by W. V. Parker, 1937, and E. W. Barankin, 1945.

The most general criterion using $R_{\mu}$ and $C_{\mu}$ was given by the inequalities

$$
R_{\mu}^{\alpha} C_{\mu}^{1-\alpha} R_{\nu}^{\alpha} C_{\nu}^{1-\alpha}<\alpha_{\mu \mu} \alpha_{\nu \nu} \quad(\mu \neq \nu ; \mu, \nu=1, \cdots, n),
$$

Received by the editors December 5, 1959; and, in revised form, March 14, 1960.

${ }^{1}$ Sponsored by the U. S. Army under contract No. DA-11-022-ORD-2059. I am indebted for discussions to Mr. Howard Bell.

2 See the note of Olga Taussky-Todd, $A$ recurring theorem on determinants, Amer. Math. Monthly vol. 56 (1949) pp. 672-676, which contains a very complete bibliography of this theorem. A fairly complete treatment of the criteria of the type considered here can be found in the book: M. Parodi, La localisation des valeurs characteristiques des matrices et ses applications, Paris, Gauthier-Villars, 1959.

' A. M. Ostrowski, Ueber das Nichtverschwinden einer Klasse von Determinanten und die Lokalisierung der charakteristischen Wurzeln von Matrizen, Compositio Math. vol. 9 (1951). This paper will be quoted in the following as U. 
where $\alpha$ is an arbitrarily chosen fixed number with

$$
0 \leqq \alpha \leqq 1 .^{8}
$$

As to $R_{\mu, \varepsilon}$ and $C_{\mu, \varepsilon,}$ the corresponding criterion is

$$
\sum_{\mu=1}^{n} \frac{1}{1+\left(\frac{\alpha_{\mu \mu}}{R_{\mu, p}}\right)^{q}}<1
$$

for a fixed but arbitrary choice of $p$ and $q$ satisfying

$$
\frac{1}{p}+\frac{1}{q}=1, \quad p \geqq 1, \quad q \geqq 1,
$$

and, of course, the criterion obtained from (7) by t eplacing $R_{\mu, p}$ by $C_{\mu, p}$. For $q=1$, we have $p=\infty$, and (7) becomes

$$
\sum_{\mu=1}^{n} \frac{1}{1+\alpha_{\mu \mu} / m_{\mu}}<1
$$

We see in particular that in the criterion (7) only $R_{\mu, 0}$ with $s \geqq 1$ are used. 4

3. In this paper we resume the method of $U$, introducing now a new parameter into the criteria using the expressions (3). Our essential result is that $D \neq 0$ if we have

$$
R_{\mu, \alpha p}^{\alpha} C_{\mu,(1-\alpha) q}^{1-\alpha}<\alpha_{\mu \mu} \quad(\mu=1, \cdots, n)
$$

for fixed but arbitrarily chosen $\alpha$ with $0<\alpha<1$ and $p$ and $q$ satisfying (6). More generally, we have the sufficient condition for $D \neq 0$ in

$$
R_{\mu, \alpha p}^{\alpha} C_{\mu,(1-\alpha) q}^{1-\alpha} R_{\nu, \alpha p}^{\alpha} C_{\nu,(1-\alpha) q}^{1-\alpha}<\alpha_{\mu \mu} \alpha_{\nu \nu} \quad(\mu \neq \nu ; \mu, \nu=1, \cdots, n) .
$$

For $p=1 / \alpha$ we obtain from (10) and (11) the criteria derived in $U$, which are of course less complicated than (10) and (11). On the other hand, using other specialization of parameters, we obtain new types of simple criteria.

4. Taking for $0<\alpha<1, q=1, p=\infty$, we obtain from (10) the conditions

$$
m_{\mu}^{1-\alpha} C_{\mu, \alpha}^{\alpha}<\alpha_{\mu \mu} \quad(\mu=1, \cdots, n),
$$

that is,

4 The criteria (7) and (9) have been derived in A. M. Ostrowski, Sur les conditions générales pour la régularité des matrices, Rend. Mat. e Appl. ser. V, vol. X (1951) pp. 156-168. 


$$
m_{\mu}^{1-\alpha} \sum_{\alpha \neq \mu} \alpha_{\alpha \mu}^{\alpha}<\alpha_{\mu \mu} \quad(\mu=1, \cdots, n),
$$

and of course the corresponding conditions obtained by using $A^{\prime}$ instead of $A$.

5. The essentially new part of the proof is that of the conditions (10), which we give in $\S \S 6,7$ as the proof of Theorem I. As to further discussions, they are in most cases exactly the same as in $U$ and we will usually be able to deal with the corresponding parts of the argument by simply referring to $U$.

6. Theorem I. $D$ does not vanish if we have, for an $\alpha$ with $0<\alpha<1$ and a couple p, $q$ satisfying (8), the inequalities (10).

Proof. Without loss of generality we can assume that $q<\infty$, since otherwise it would be sufficient to apply the discussion to $A^{\prime}$.

If we had $D=0$, we would have a nontrivial solution $\left(x_{1}, \cdots, x_{n}\right)$ of the system

$$
\sum_{\nu=1}^{n} \alpha_{\mu \nu} x_{\nu}=0 \quad(\mu=1, \cdots, n) .
$$

Put

$$
\left|a_{\mu \nu}\right|=\alpha_{\mu \nu}, \quad\left|x_{\nu}\right|=\xi_{\nu} \quad(\mu, \nu=1, \cdots, n) .
$$

Then, isolating in (13) the term with $x_{\mu}$ and going over to the moduli, we obtain

$$
\alpha_{\mu \mu} \xi_{\mu} \leqq \sum_{\nu \neq \mu} \alpha_{\mu \nu} \xi_{\nu} \quad(\mu=1, \cdots, n) ;
$$

this can be written in the form

$$
\alpha_{\mu \mu} \xi_{\mu} \leqq \sum_{\nu \neq \mu} \frac{\alpha}{\alpha_{\mu \nu}\left(\alpha_{\mu \nu}^{1-\alpha} \xi_{\nu}\right)} \quad(\mu=1, \cdots, n) .
$$

Applying to (16) Hölder's inequality, we obtain

$$
\alpha_{\mu \mu} \xi_{\mu} \leqq\left(\sum_{\nu \neq \mu} \alpha_{\mu \nu}^{\alpha p}\right)^{1 / p}\left(\sum_{\nu \neq \mu} \alpha_{\mu \nu}^{(1-\alpha) q} \xi_{\nu}^{q}\right)^{1 / q} \quad(\mu=1, \cdots, n),
$$

or using (3),

$$
\alpha_{\mu \mu} \xi_{\mu} \leqq R_{\mu, \alpha p}^{\alpha}\left(\sum_{\nu \neq \mu} \alpha_{\mu \nu}^{(1-\alpha) q} \xi_{\nu}^{q}\right)^{1 / q} \quad(\mu=1, \cdots, n) .
$$

7. Raising (17) to the $q$ th power, we can write 


$$
R_{\mu, \alpha p}^{-\alpha q} \alpha_{\mu \mu}^{q} \xi_{\mu}^{q} \leqq \sum_{\nu \neq \mu} \alpha_{\mu \nu}^{(1-\alpha) q} \xi_{\nu}^{q} \quad(\mu=1, \cdots, n) .
$$

Summing this over $\mu=1, \cdots, n$, we obtain

$$
\sum_{\mu=1}^{n} R_{\mu, \alpha p}^{-\alpha q} \alpha_{\mu \mu}^{q} \xi_{\mu}^{q} \leqq \sum_{\mu=1}^{n} \sum_{\nu \neq \mu} \alpha_{\mu \nu}^{(1-\alpha) q} \xi_{\nu}^{q}
$$

But, if we interchange the order of summation in the right-hand sum and use (3), we obtain

$$
\sum_{\nu=1}^{n} \xi_{\nu}^{q} \sum_{\mu \neq \nu} \alpha_{\mu \nu}^{(1-\alpha) q}=\sum_{\nu=1}^{n} C_{\nu,(1-\alpha) q}^{(1-\alpha) q} \xi_{\nu}^{q}=\sum_{\mu=1}^{n} C_{\mu,(1-\alpha) q}^{(1-\alpha) q} \xi_{\mu \nu}^{q}
$$

and we get

$$
\sum_{\mu=1}^{n} R_{\mu, \alpha p}^{-\alpha q} \alpha_{\mu \mu}^{q} \xi_{\mu}^{q} \leqq \sum_{\mu=1}^{n} C_{\mu,(1-\alpha) q}^{(1-\alpha) q} \xi_{\mu .}^{q}
$$

But, since at least one of $\xi_{\mu}^{q}$ is unequal to zero, (18) cannot hold if we have (10). Theorem I is proved.

8. As in $U$, we can replace the right-hand expressions in the inequalities (10) by

$$
\alpha R_{\mu, \alpha p}+(1-\alpha) C_{\mu,(1-\alpha) q}
$$

as we have generally

$$
\alpha x+(1-\alpha) y \geqq x^{\alpha} y^{1-\alpha} \quad(x>0, y>0,0 \leqq \alpha \leqq 1) .
$$

In this way we obtain a weaker formulation of Theorem 1, which is, however, sometimes easier to apply numerically.

From Theorem I follows immediately

Theorem II. $D$ does not vanish if we have for an $\alpha$ with $0<\alpha<1$ and a couple $p, q$ satisfying (8) the inequalities (11).

The proof is literally the same as in $U, \S 19$, if we introduce the expressions

$$
s_{\mu}=\frac{1}{\alpha_{\mu \mu}} R_{\mu, \alpha p}^{\alpha} C_{\mu,(1-\alpha) q}^{1-\alpha}
$$

9. An immediate consequence of Theorem 1 is

THEOREM III. For any $\alpha$ with (6) and any couple $p, q$ satisfying (8) each fundamental root of $A$ lies inside or on one of the circles 


$$
\left|\lambda-\alpha_{\mu \mu}\right| \leqq R_{\mu, \alpha p}^{\alpha} C_{\mu,(1-\alpha) q}^{1-\alpha} \quad(\mu=1, \cdots, n) .
$$

The modulus of each fundamental root of $A$ is at the most equal to

$$
\underset{\mu}{\operatorname{Max}}\left(\alpha_{\mu \mu}+R_{\mu, \alpha p}^{\alpha} C_{\mu,(1-\alpha) q}^{1-\alpha}\right) \leqq\left(\sum_{k=1}^{n} \alpha_{\mu p}\right)^{1 / p}\left(\sum_{k=1}^{n} \alpha_{k \mu}^{(1-\alpha) q}\right)^{1 / q} .
$$

The inequality in (23) follows, of course, from Hölder's inequality.

Particularly simple expressions are obtained if we consider the limiting case $q=1$. Then (23) becomes

$$
\underset{\mu}{\operatorname{Max}}\left(\alpha_{\mu \mu}+m_{\mu}^{\alpha} \sum_{\alpha \neq \mu} \alpha_{\kappa \mu}^{1-\alpha}\right) .
$$

Define

$$
M_{\mu}=\underset{\mu}{\operatorname{Max}}\left(\alpha_{\mu \mu}, m_{\mu}\right)=\underset{\kappa}{\operatorname{Max}} \alpha_{\mu \kappa} .
$$

Then we have as a bound for the moduli of all fundamental roots of $A$

$$
\underset{\mu}{\operatorname{Max}} M_{\mu}^{\alpha} \sum_{\alpha=1}^{n} \alpha_{\kappa \mu}^{1-\alpha} \text {. }
$$

Similarly, from Theorem II it follows that for any choice of $\alpha, p, q$ satisfying (6) and (8) each fundamental root of $A$ lies inside or on the boundary of one of the ${ }_{n} C_{2}$ lemniscate-shaped domains

$$
\left|\lambda-\alpha_{\mu \mu}\right|\left|\lambda-\alpha_{\nu \nu}\right| \leqq\left(R_{\mu, \alpha p} R_{\nu, \alpha p}\right)^{\alpha}\left(C_{\mu,(1-\alpha) q} C_{\nu,(1-\alpha) q}\right)^{1-\alpha}(\mu \neq \nu) .
$$

10. In the case of Hadamard's theorem, O. Taussky-Todd generalized the argument to the case where the $>$ in the inequalities (1) are replaced by $\geqq$. The corresponding discussion for the criterion using simultaneously $R_{\mu}$ and $C_{\mu}$ was given in $\mathrm{U}$ as Theorems VI and VII and proved in $\$ 11-18$. Exactly the same argument as in U can be applied to the inequalities (10) and the inequalities using (19). We obtain

TheOREM IV. Assume an $\alpha$ with $0<\alpha<1$ and a couple $p, q$ satisfying (8) with $p<\infty, q<\infty$.

If we have for the matrix $A$ the inequalities

$$
R_{\mu, \alpha p}^{\alpha} C_{\mu,(1-\alpha) q}^{1-\alpha} \leqq \alpha_{\mu \mu} \quad(\mu=1, \cdots, n),
$$

and if $D=0$, then either $A$ is totally reducible or we have in all relations (27) the equality sign and $A$ is irreducible.

If we have 


$$
\alpha R_{\mu, \alpha p}+(1-\alpha) C_{\mu,(1-\alpha) q} \leqq \alpha_{\mu \mu} \quad(\mu=1, \cdots, n),
$$

if the matrix is totally irreducible and if $D=0$, then the equality sign holds in all relations (28), we have for each $\mu: R_{\mu, \alpha p}=C_{\mu,(1-\alpha) q}$ and $A$ is simply irreducible. In this case the rows and columns of $A$ can be multiplied by convenient factors of modulus 1 in such a way that after this multiplication the sums of the elements in each row and in each column vanish. ${ }^{5}$

11. It may finally be observed that from the results proved in a previous communication ${ }^{6}$ and from Theorem I and Theorem II follows

Theorem V. Under the conditions of Theorem I and Theorem II, the columns of $A$ can be multiplied by convenient positive factors in such $a$ way that after this multiplication the relations (1) hold.

- A matrix $A$ is reducible if by a cogradient permutation of rows and columns it can be brought into the form $\left(\begin{array}{ll}P & 0 \\ R & Q\end{array}\right)$; otherwise, it is irreducible. $A$ is totally reducible if by a cogradient permutation of rows and columns it can be brought into the form $\left(\begin{array}{ll}P & 0 \\ 0 & Q\end{array}\right)$; otherwise, $A$ is totally irreducible.

- Alexander Ostrowski, Über die Determinanten mit überwiegender Hauptdiagonale, Comment. Math. Helv. vol. 10 (1937) pp. 69-96.

Mathematics Research Center of the U. S. Army 